tion of 0.82 per cent concentration has a relative viscosity of 52 ; on addition of potassium chloride to $0 \cdot 1 M$ the viscosity falls to a value of 3.3 and fiow-birefringence disappears. Both in absence and presence of salts the protein precipitates at $p \mathbf{H} \quad 4 \cdot 5-5$ and redissolves below $p \mathrm{H} 4 \cdot 2$. Its solubility above $p \mathrm{H} 6$ is not affected by heat treatment at sny $p \mathrm{H}$, nor is it rendered insoluble by organic solvents, strong urea solutions or trichloroacetic acid.

The flbrous character of the protein is indicated by the high frictional ratio of $3 \cdot 1$, the high viscosity, and the $X$-ray investigations carried ratio of $3 \cdot 1$, the high viscosity, and the $\mathrm{X}$-ray investigations carried
out by Prof. W. T. Astbury and Mr. T. C. Spark. These latter reveal that tropomyosin belongs to the keratin-myosin-flbrinogen group. $A$ dried fllm photographed with the $\mathrm{X}$-ray beam perpendicular to the A dried flm photographed with the X-ray beam perpendicular to the
Burface gives a disoriented pattern of the $\alpha$-type in which the $5 \cdot 1 \mathrm{~A}$. spacing is unusually clear and strong, while the same film photographed with the beam parallel to the surface gives a partially oriented fibre wattern. The $\alpha$-fold appears to survive treatments (such as stretching pattern. The $a$-fold appears to survive treatments (such as stretching of squeezing in the cold moist state) which generally bring about the $\alpha-\beta$ transformation, but it is found that a strong $\beta$-pattern appears on heating to $105^{\circ}$ or on squeezing between pieces of plate-glass that have
first been heated in steam: and in the $\beta$-films produced by the latter fritheen heated in steam ; and in the $\beta$-films produced by the latter method, not only do the main chains tend to lie parallel to the flum, but also the side chains tend to stand perpendicula

as has been observed with keratin and myosin. Tropomyosin contains no phosphorus, carbohydrate or hexosamine.
Its amino-acid composition resembles that of myosin itself, especially with respect to the monoamino-monocarboxylic acids, determined chromatographically by Dr. G. R. Tristram. Differences are found in the high lysine nitrogen (18 per cent of the protein nitrogen as compared with 12.6 per cent for myosin), in the lower amide nitrogen ( 4.94 per cent, as against 7.2 per cent) and in the complete absence of tryptophan. The dicarboxylic acids, as yet undetermined, must be equivalent to the total base plus amide-nitrogen since the isoelectric point is in the acid $p \mathrm{H}$ range. It seems probable indeed that tropomyosin is unique among proteins in carrying the highest known valence both of positive and negative type. 'This property is obviously related to the aggregation phenomena implied by the high viscosity of tropomyosin in absence of salt.

The function of tropomyosin is as yet unknown. Since it is not present in the sarcoplasm it would appear to be associated with the fibrl itself, where it is firmly bound either to the myosin or to some other structural component or to both. Though water-soluble after isolation, it is only extracted by salt solutions from ethanol-treated muscle, and without the latter treatment only partial extraction is muscle, and without the later the facts suggest that in situ it forms an insoluble complex possible. These facts suggest that in situ it forms an insoluble complex liberated by a process of metathesis in presence of salt. The amount liberated by a process of metathesis in presence of salt. The amount extracted is a linear function of salt concentration up to $\mu=1$, and
thereafter is maximal. The yield from rabbit skeletal muscle is $0.5 \mathrm{gm} . / 100 \mathrm{gm}$. wet muscle weight, and rather more (1 per cent) in the case of very young a nimals.

The exact relation of tropomyosin to myosin itself is equally obscure, but the analytical and structural similarities indicate that it is a species of myosin differing mainly in the length of the polypeptide chain. In proposing the present name, we have deemed it desirable to retain the word 'myosin' and to add a prefix which suggests this speciflc relationship. Experiments have shown that tropomyosin is
not derived from myosin itself by the disaggregation of denatured not derived from myosin itself by the disaggregation of denatured myosin in presence of salts, nor by any kind of post-mortem catheptic activity. 'Purifled' myosin (that is, three times precipitated) itself contains about $0 \cdot 1$ per cent of tropomyosin, but the association is it is responsible for the adenosinetriphosphatase activity of the myosin complex. It may conceivably be a precursor of myosin, a fragmentar chain used in the elaboration of the gigantic myosin molecule. It should be noted that the protein is unrelated to the actin preparations of Straubi , for which no criteria of homogeneity have been advanced; und tropomyosin, these are susceptible to denaturing processe of salt.

Department of Biochemistry,

KENNETH BAILEY.

Cambridge. Jan. 11.

${ }^{1}$ Straub, F. B., Stud. Inst. Med. Chem. Univ. Szeged, 3, 23 (1943).

\section{Absence of Pseudo-Cholinesterase from the Tissues of} Ruminants In a previous paper ${ }^{1}$ it was mentioned that the non-specifle or
pseudo-cholinesterase is absent from the blood and tissues of ox and sheep. Although the experiments with blood were conclusive ${ }^{2}$, an observation, difficult to interpret at that time, was made with one of the tissues examined, namely, beef kidney. This tissue was found to be capable of hydrolysing benzoylcholine, a compound suggested by Mendel, Mundell and Rudney ${ }^{2}$ as a substrate suitable for measuring the activity of pseudo-cholinesterase. The activity towards this substrate, however, proved to be resistant to a concentration of eserine which inhibits the pseudo-cholinesterase in other mammalian tissues a fact which made it appear unlikely that the enzyme in beef kidney was a pseudo-cholinesterase.

Sawyer's demonstration of the existence of an esterase capable of hydrolysing benzoylcholine ${ }^{3}$ offers an adequate explanation for this discrepancy. Benzoyleholine esterase, which, according to Sawyer, is present in the liver of certain rodents, hydrolyses benzoylcholine but not acetylcholine. Recent experiments in this laboratory ${ }^{4}$ have shown that benzoylcholine esterase is not inhibited by $2 \times 10^{-8} M$ eserine, a concentration which inhibits the activity of pseudo-cholinesterase. Experiments based on this flnding reveal that the hydrolysis of benzoylcholine by beef kidney is due to benzoylcholine esterase, since the activity of this tissue towards benzoylcholine is insensitive to eserine in the above-mentioned concentration.

Other tissues of ox and sheep which, by their inability to hydrolyse benzoylcholine, were shown to contain no pseudo-cholinesterase are liver, spleen, pancreas, muscle (Masseter), gastric mucosa, intestinal mucosa, thyroid gland, parotid gland, adrenal gland, sublingual gland, lachrymal gland.

The fact that pseudo-cholinesterase, previously shown to be absent from brain tissue throughout the animal kingdom ${ }^{5}$, has now been found to be absent from the tissues of ox and sheep, is a further indication of the irrelevance of this enzyme to the process of nerve impulse transmission.

Josephine M. GUNTER. Medical Research,

University of Toronto, Toronto. Nov. 1.

${ }^{1}$ Mendel, B., and Rudney, H., Science, 100, 499 (1944).

'Mendel, B., Mundell, D. B., and Rudney, H., Biochem. J., 37, 473 (1943).

${ }^{3}$ Sawyer, C. H., Science, 101, 385 (1945)

Mendel, B., and Gunter, J. M., unpublished experiments.

Mendel, B., and Rudney, H., Science, 98, 201 (1943).

A Substance with Sympathin E Properties in Spleen Extracts

ALCOHOLIC extracts of fresh spleen from cattle contain high amounts, up to $10 \mu \mathrm{gm}$. adrenaline equivalents per gm. tissue, of a cardioaccelerator and blood-pressure raising substance, showing the characteristic properties of a sympathomometic catechol compound ${ }^{1}$. The active substance was taken up in an ether solution of organ lipids and extracted from this with a 5-10 per cent solution of sodium sulphate. A further purification was attained by treatment with sublimate in alcohol, which left the active substance in the flitrate. The purifled extract was found to differ from adrenaline in the following respects. Ergotamine, in a dose which inhibited or reversed the ing respects. Ergotamine, in a dose which inhibited or reversed the action of adrenaline, was not equally effective in inhibiting the pressor non-pregnant cat was rather less inhibited than bv an eguipressor non-pregnant cat was rather less inhibited than by an eguipressor dose of adrenaline, and the same applied to the isolated intestine of
the rabbit. The relative dilating effect on the iris was considerably the rabbit. The relative dilating effect on the iris was considerably and the spleen pressor substance gave similar catechol reactions with ferric chloride, the fluorescence reaction in alkaline solution ${ }^{2}$ was absent in purifled spleen extracts. The active substance in spleen thus conforms better with an amino-base, such as nor-adrenaline, thas conforms better
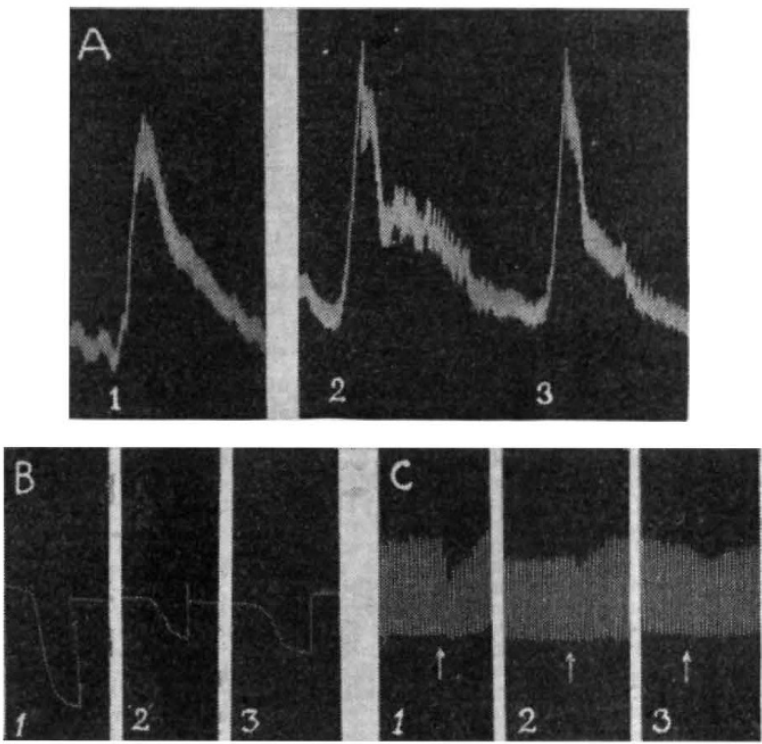

$A$, BLOOD PRESSURE, CAT; $B$, ISOLATED VIRGIN CAT'S UTERUS ; $C$, ISOLATED RABBIT'S JEJUNUM; $; 1,2, \mu \mathrm{GM}$. ADRENAIINE;
$2,0 \cdot 1$ PURIFIED SPLEEN EXTRACT; $3,2 \mu \mathrm{GM} .3,4$-DIHYDROXY-nor-

Direct comparison of equipressor doses of adrenaline (I), 3,4-dihydroxy-nor-ephedrine (II) and spleen extracts (III) with regard to biological tests, colour and fluorescence reactions gave much closer agreement between (II) and (III) than between (I) and (III) (see figure). Dioxy-nor-ephedrine, on the other hand, is similar in action to the amino-base nor-adrenaline, which in its turn closely resembles the effect of stimulation of certain adrenergic nerves ${ }^{3}$. The good agreement between the action of purified spleen extracts, nor-adrenaline and the mediator of hepatic nerve stimulation suggests, flrst, that the active substance in spleen is closely related to nor-adrenaline. and, secondly, that the spleen aubstance is, in fact, the postulated sympathin E.

\section{Physiology Department,}

U. S. v. EULER.

Karolinska Institutet,

Euler, U. S. v., Nature, 156, 18 (1945).

' Gaddum, J. H., and Sehild, H., J. Physiol., 80, 9 P (1934)

3 Barger, G., and Dale, H. H., $J$. Physiol., 41, 19 (1910).

(10n, . B., and Rosenblueth, A., Amer. J. Physiol., 104, 557

(1933). Greer, C. M., Pinkston, J. O., Baxter, J. H., and Brannon,
E. S., J. Pharm. Exp. Ther., 62, 189 (1935). 